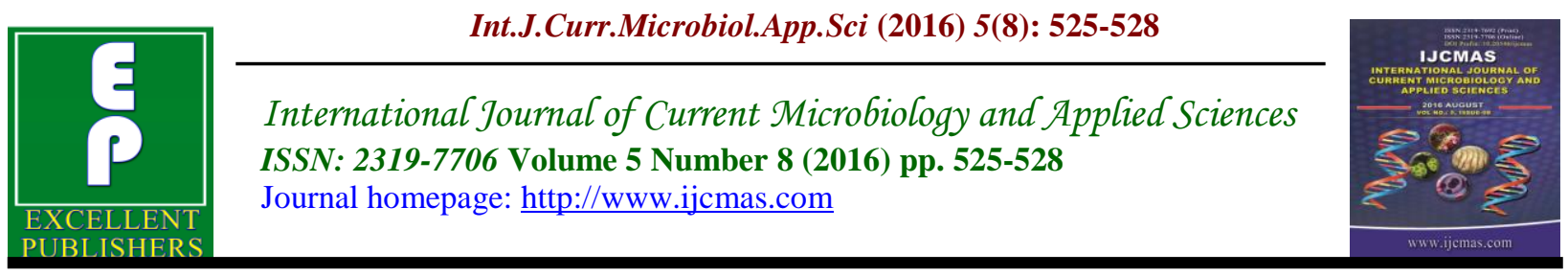

Original Research Article

http://dx.doi.org/10.20546/ijcmas.2016.508.056

\title{
Isolation and Sensitivity Pattern of Genital Candida Species in STD OP Female Attendees of Tertiary Care Hospital, Chennai, India
}

\author{
R. Vanaja ${ }^{1 *}$, V. Sudha ${ }^{2}$ and Mangala Adisesh ${ }^{3}$ \\ ${ }^{1}$ Department of Microbiology, Madras Medical College, Chennai, India \\ ${ }^{2}$ Department of Dermatology, ACS medical college, Chennai, India \\ ${ }^{3}$ Department of Microbiology, MMC \& RGGGH, Chennai, India \\ *Corresponding author
}

\begin{abstract}
A B S T R A C T
Keywords

Vaginal discharge,

Candida,

Hichrome agar,

Nystatin and

Micanazole.

Article Info

Accepted:

23 July 2016

Available Online:

10 August 2016

The study was aimed to isolate Candida species from vaginal discharge of female patients with complaints of itching and to determine susceptibility pattern of vaginal isolates of Candida to antifungal drugs. A total of 400 vaginal discharge samples from all the female patients attending STD OP, Institute of Venereology, RGGGH / Madras Medical College, Chennai were included in this study. All the samples were subjected to KOH mount, cultured on Sabouraud's Dextrose Agar, incubated overnight at room temperature, further gram stain and germ tube test were performed and subcultured on Hichrome Candida differentiation agar (M1297A). The species were differentiated by the morphology and colour of colonies. Antifungal susceptibility testing was done for all the species. Out of 400 samples, 83 patients had Candida isolations: C.glabrata - cream coloured colonies $37(45 \%)$, C.albicans - green coloured colonies 25(30\%), C.tropicalis - blue coloured colonies 14(17\%), C.krusei - pink coloured colonies 7(8\%). All 83 isolates showed sensitivity to miconazole and nystatin, 39 to itraconazole, 6 to clotrimazole, 1 to fluconazole and nil with ketoconazole.
\end{abstract}

\section{Introduction}

Fungal infections have been increasingly reported worldwide. The emergence of fungal infections has been attributed to advancement in medical management and increasing load of severely ill patients. The last few decades have seen a steady increase in the incidence of fungal infections especially Candida species.

Candida species are normal commensals of human skin, throat, gastrointestinal tract, female genital tract and urinary tract with catheters. Candida is one of the common opportunistic fungal infections.

Candidiasis is the most common fungal disease with wide spectrum of clinical presentation. The infections may be acute or chronic, superficial or deep involving various systems.

Vaginal candidiasis is a common problem for women and may affect their physical and emotional health. Several predisposing 
factors such as diabetes mellitus, uterine contraceptives, pregnancy and broad spectrum antibiotics are reported as risk factors for candidial infection. Vulvovaginal candidiasis remains a common cause of morbidity among adult women. While the main etiological agents of vaginal candidiasis is being changing from C.albicans to other non-albicans species, Knowledge of identification of patterns of genital Candida species and drug resistant candidial isolates is important for management.

The present study was carried out to isolate the species and test the susceptibility pattern of Candida isolates from the vaginal discharge.

\section{Materials and Methods}

A total of 400 vaginal discharge samples were collected from all the female patients attending STD OP, Institute of Venereology, RGGGH/ Madras Medical College, Chennai. The swabs were subjected to $\mathrm{KOH}$ mount, cultured on Sabouraud's Dextrose Agar and incubated at room temperature overnight. SDA culture showing confluent heavy growth with cream colored, pasty colonies suggesting Candida were further characterized. Gram stain and germ tube test were done for all the isolates. The colonies were further subcultured on to Hichrome agar and incubated at room temperature $\left(25^{\circ} \mathrm{C}\right)$ overnight. The plates were further incubated for 48 hours to get better developed colored colonies. Presumptive identification of Candida species was made by distinctive color and morphology of the colonies as per the manufacturer's instructions. The antifungal susceptibility testing was done on SDA by disc diffusion method (Zahra Salehei et al., 2012). A suspension of overnight cultures of Candida isolates was prepared in sterile normal saline. The suspension was inoculated on
SDA plates and rolled on the surface of the agar medium. The plates were dried for 15 minutes at room temperature in laminar hood and then the antifungal discs were placed on the inoculated agar with sterile forceps. The plates were incubated at room temperature for 24 hours and zone diameters were measured manually [Table 3].

The antifungal discs used were Nystatin 100 units/disc, Miconazole50 $\mu \mathrm{g}$, Itraconazole $10 \mu \mathrm{g}$, Clotrimazole 50 $\mathrm{\mu g}$, Fluconazole/ Ketoconazole $10 \mu \mathrm{g}$ each (Hi Media).

\section{Results and Discussion}

In the present study, among 400 samples $83(21 \%)$ had candidiasis. These clinical isolates namely C.glabrata - cream coloured $37(45 \%)$, C.albicans - green coloured 25(30\%), C.tropicalis - blue coloured 14(17\%) and C.krusei - pink coloured $7(8 \%)$ colonies were identified (table 1). Some isolates showed pseudo hyphae and yeast cells, and some isolates showed only yeast cells without pseudo hyphae. There were few differences in microscopic morphology of yeast cells. C.tropicalis and C.krusei showed large ovoid yeast cells. C.albicans and C.glabrata species showed round budding yeast cells.

All the isolates were sensitive to miconazole and nystatin. 39 isolates showed sensitivity to itraconazole, 6 to clotrimazole, 1 to fluconazole and none to ketoconazole. (Table 2).

For C.albicans, out of 25/83 isolates 1 isolate showed sensitivity to fluconazole, 25 showed sensitivity to miconazole and nystatin, 16 to itraconazole and 4 to clotrimazole.

All the C.glabrata isolates $37 / 83$ showed sensitivity to miconazole and nystatin, 2 for clotrimazole and 13 for itraconazole. 
(Figure.1). All the 14/83 isolates of C.tropicalis showed sensitivity to miconazole and nystatin and 7 isolates to itraconazole. None of the isolates were sensitive to ketoconazole, fluconazole and clotrimazole.

Table.1 Candida speciation using Hi Chrome Candida differentiation media

\begin{tabular}{|l|l|l|l|l|}
\hline S.NO & $\begin{array}{l}\text { NAME OF THE } \\
\text { SPECIES }\end{array}$ & COLOUR & $\begin{array}{l}\text { NO.OF } \\
\text { ISOLATES }\end{array}$ & $\%$ \\
\hline 1 & C.glabrata & Cream & 37 & $45 \%$ \\
\hline 2 & C.albicans & Green & 25 & $30 \%$ \\
\hline 3 & C.tropicalis & Blue & 14 & $17 \%$ \\
\hline 4 & C.krusei & Pink & 7 & $8 \%$ \\
\hline
\end{tabular}

Table.2 The sensitivity pattern of Candida species to various antifungal agents.

\begin{tabular}{|c|c|c|c|c|c|c|c|}
\hline \multirow[t]{2}{*}{ S.No } & \multirow{2}{*}{$\begin{array}{l}\text { CANDIDA } \\
\text { SPECIES }\end{array}$} & \multicolumn{6}{|c|}{ ANTIFUNGAL SENSITIVITY } \\
\hline & & MIC & NYS & ITR & FLU & CLO & KET \\
\hline 1 & C.glabrata & 37 & 37 & 13 & 0 & 2 & 0 \\
\hline 2 & C.albicans & 25 & 25 & 16 & 1 & 4 & 0 \\
\hline 3 & C.tropicalis & 14 & 14 & 7 & 0 & 0 & 0 \\
\hline 4 & C.krusei & 7 & 7 & 3 & 0 & 0 & 0 \\
\hline \multicolumn{2}{|c|}{ TOTAL } & 83 & 83 & 39 & 1 & 6 & 0 \\
\hline
\end{tabular}

Fig.1 The antifungal susceptiblity pattern of Candida species.

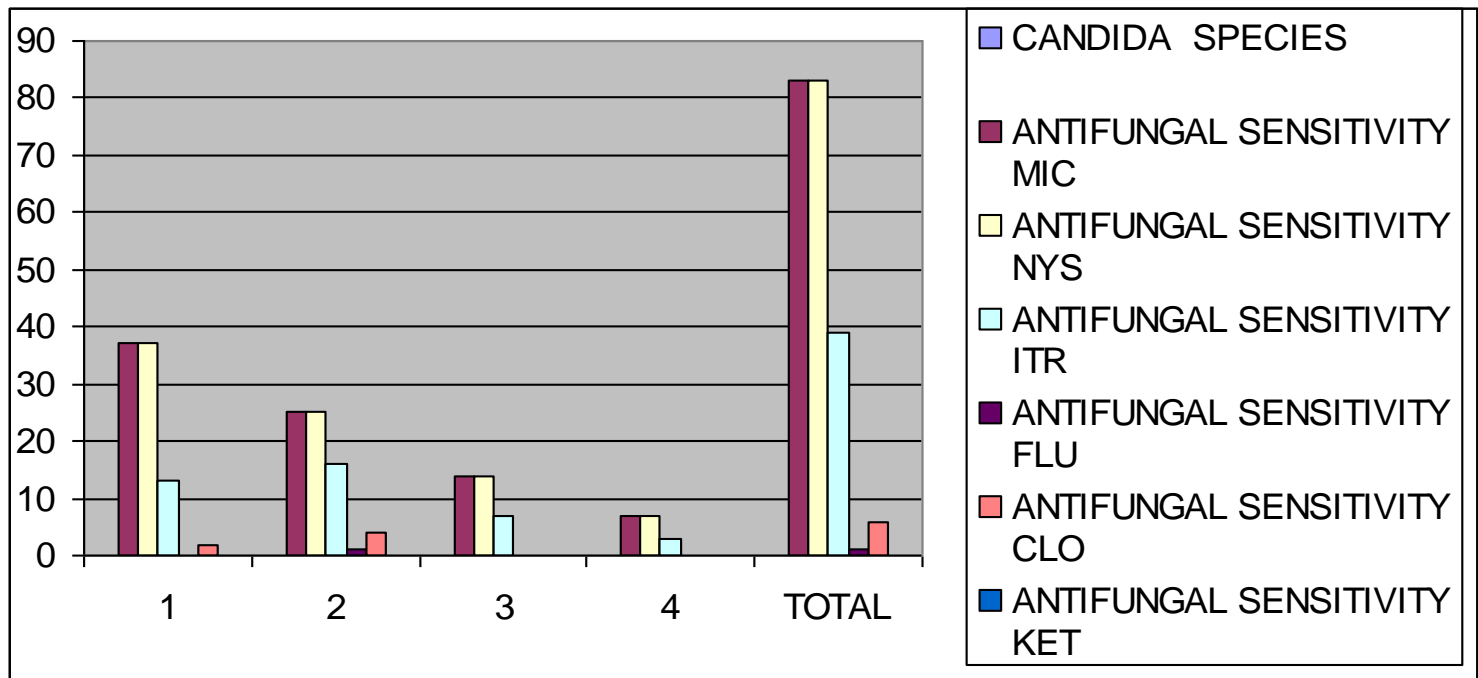

Out of 7/83 isolates of C.krusei, all 7 showed sensitivity to miconazole and nystatin and 3 to itraconazole. Candidial vaginitis is a common fungal infection among adult women during reproductive age. Fortunately, the infection is rarely life threatening but usually associated with morbidities like discomfort, pain, sexual dysfunctions, vulval dryness, cracks, itching, burning, soreness and finally health care cost (Paulitsch et al., 2006).

Earlier studies found that C.albicans, the most common isolate as a main agent of vaginal candidiasis (Richter et al., 2005). However; it seems that non albicans species 
(C.glabrata, C.tropicalis, C.krusei) appear to be in increase (Defontaine et al., 1999). This is correlating with our study. In other studies C.krusei and C.glabrata of Candida species appear to be increasing. However (Mohanty et al., 2007) has reported C.glabrata as the main etiology of VVC which is correlating with our study.

Clotrimazole and Fluconazole are the two antifungal drugs widely used in the treatment of VVC. The sensitivity patterns of Candida isolates varies among studies in different countries. Quindos et al., (1999) study showed that the Candida species were sensitive to Fluconazole and Ketoconazole. Zahra Seifi et al., (2012) study showed Candida species were resistant to Fluconazole. Our results showed that the Candida isolates were resistant to Fluconazole and Ketoconazole. This study indicated that $C$. glabrata was the predominant isolate and that the drug pattern are changing. Fluconazole the systemic drug of choice for genital candidiasis is less sensitive and resistant whereas Miconazole and Nystatin are highly sensitive.

Further studies are required for confirming the drug sensitivity pattern, so that appropriate antifungal drugs could be selected to manage candidiasis and to prevent its recurrence and the morbidity in the patients. Further critical studies with large sample size are needed.

\section{References}

Defontaine, A., Bouchara, J.P., Declerk, P., Planchenault, C., Chabasse, D., Hallet,
J.N. 1999. Invitro resistance to azoles associated with mitochondrial DNA deficiency in Candida glabrata, J. Med. Microbiol., 48(7): 663-70. DOI (PubMed)

Mohanty, S., Xess, I., Hassan, F., Kapil, A., Mittal, S., Tolosa, J.E. 2007. Prevalence and susceptibility to fluconazole of Candida species causing vulvovaginitis. Indian J. Med. Res., 126(3): 216-9. (PUBMed)

Paulitsch, A., Wager, W., GinterHanselmayer, G., Marth, E., Buzina, W. 2006. A-5-year (2000-2004) epidemiological survey of Candida and non-Candida yeast species causing vulvovaginal candidiasis in Graz, Austrua Mycoses, 49(6): 471-5. (DOI) (PubMed).

Quindos, G., Abarca, L., Carrillo-Munoz, A.J., Arevalo, M.P., Bornay, F.J., Casals, J.B., et al. 1999. Multicenter sorvey of in vitro antifungal resistance in yeast of medical importante isolated from Spanish patients, Rev. Iberoam. Micol., 16(2): 97-100. (PubMed)

Richter, S.S., Galask, R.P., Messer, S.A., Hollis, R.J., Diekema, D.J., Pfaller, M.A. 2005. Antifungal Susceptibilities of Candida Species causing vulvovaginitis and epidemiology of recurrent cases, J. Clin Microbiol., 43(5): 2155-62. DOI (PubMed)

Zahra Salehei, Zhra Seifi, and Ali Mahmoudabadi, et al. 2012. Sensitivity of vaginal isolates of Candida to Eight Antifungal Drugs isolated from Ahvaz, Iran. Jundishapur J. Microbiol., 5(4): 574-7. DOI:10.5812/jjm.4556

\section{How to cite this article:}

Vanaja, R., V. Sudha and Mangala Adisesh. 2016. Isolation and Sensitivity Pattern of Genital Candida Species in STD OP Female Attendees of Tertiary Care Hospital, Chennai, India. Int.J.Curr.Microbiol.App.Sci. 5(8): 525-528. doi: http://dx.doi.org/10.20546/ijcmas.2016.508.056 